\title{
Unmanned Aerial Vehicle Systems for Disaster Relief: Tornado Alley
}

\author{
Wesley M. DeBusk* \\ Georgia Institute of Technology, Atlanta, GA
}

\begin{abstract}
Unmanned aerial vehicle systems are currently in limited use for public service missions worldwide. Development of civil unmanned technology in the United States currently lags behind military unmanned technology development in part because of unresolved regulatory and technological issues. Civil unmanned aerial vehicle systems have potential to augment disaster relief and emergency response efforts. Optimal design of aerial systems for such applications will lead to unmanned vehicles which provide maximum potentiality for relief and emergency response while accounting for public safety concerns and regulatory requirements. A case study is presented that demonstrates application of a civil unmanned system to a disaster relief mission with the intent on saving lives. The concept utilizes unmanned aircraft to obtain advanced warning and damage assessments for tornados and severe thunderstorms. Overview of a tornado watch mission architecture as well as commentary on risk, cost, need for, and design tradeoffs for unmanned aerial systems are provided.
\end{abstract}

\section{Introduction}

Modern disaster relief and emergency response (DRER) services in the United States are provided by networks of law enforcement, fire protection, military, and other special service personnel. The concept of implementing unmanned aerial vehicle (UAV) systems to assist DRER efforts has been previously proposed ${ }^{1}$. This paper will expand upon these ideas into the use of civil-sector UAV systems to aid DRER efforts. Emphasis will be placed on analysis of technology requirements and system characteristics. A case study on a notional application of UAV systems in "tornado alley" -- storm and tornado tracking, warning, and relief response -- will be presented as a demonstration of analysis techniques.

\section{A. Civil UAV Status}

Unmanned aerial vehicles (UAV) are currently used in a variety of civil applications. The military market currently dominates the unmanned systems sector largely due to significantly fewer operational constraints. Strict airspace restrictions, underdeveloped technology, and lack of funding and support are the primary barriers to the growth of the civil UAV market in the United States. Limited examples of market barrier breakthroughs and growth exist, however as a whole the civil UAV community has been slow to capitalize on these early promising endeavors.

\section{B. Civil UAV Missions}

Civil uses for UAV systems can be categorized into four general categories ${ }^{21}$ : Land Management, Earth Science, Homeland Security, and Commercial. Land management systems are related to the observation and exploration of land for the purpose of tracking, monitoring, mapping, surveying, or other related tasks. Such missions include forest fire tracking, crop monitoring and spraying, and wildlife herd tracking. Earth science includes all systems designed to gather and interpret data intended for scientific research. Weather tracking, remote aerial surveying, and habitat monitoring are examples of earth science missions. Homeland security systems assist law enforcement, monitor borders, and provide emergency response services. Commercial systems are those used by corporations or for private ventures. This is a

\footnotetext{
* Education Associates Program Intern, Aeromechanics Branch, Flight Vehicle Research and Technology
} Division, Mail Stop 243-10, NASA Ames Research Center, Moffett Field, CA, 94035-1000. 
broad category and examples of missions range from aerial video and photography to pipeline and transmission line monitoring to wireless communications.

It could be argued that a fifth category exists which encompasses public service missions. Disaster relief and emergency response missions, which are in actuality split between other categories, would fall into this public service category. Public service systems would include those that interface with public civil services, such as highway patrol, traffic routing, and utilities monitoring.

\section{Operational Civil UAV Platforms}

Several operational civil UAV platforms currently exist. Many have been adapted to civil use though originally designed for military use (e.g. Predator). Other platforms were initially designed for specific civil uses and have later been converted to more general use (e.g. RMAX). Platforms compatible with unmanned aerial DRER missions currently exist, however new or modified/enhanced platforms will likely need to be developed. Below are descriptions of several UAV platforms currently operated by NASA for civil missions.

Aerosonde is a currently operational civil UAV platform originally designed in Australia for meteorological reconnaissance and environmental monitoring. NASA Goddard Space Flight Center operates Aerosondes for earth science missions.

Altair is a currently operational civil UAV platform designed by General Atomics. It was designed as a high altitude more reliable version of the Predator B. It is currently operated by General Atomics and NASA Dryden Flight Research Center.

Ikhana is a currently operational civil UAV platform designed by General Atomics. It was designed as a civil variant of the Predator B. NASA currently operates the Ikahana at Dryden Flight Research Center for civil missions.

Yamaha RMAX helicopter is a currently operational civil UAV platform which is widely used around the world. It was originally designed for agricultural and surveillance purposes, though it has been used for many other purposes. A NASA/U.S. Army AFDD collaborative currently uses an RMAX helicopter for autonomous DRER-type surveillance ${ }^{22}$.

\section{Disaster Relief and Emergency Response Mission}

Disaster relief and emergency response (DRER) efforts are inclusive of all actions by first responders and subsequent aid efforts during and immediately following a catastrophic event which threatens human life. The primary purpose of DRER efforts is to save human lives. Secondary purposes of DRER efforts are to preserve and maintain the environment, protect property, keep the peace, and uphold governmental authority.

Modern manned DRER systems include ground vehicles, conventional aircraft, and rotorcraft ${ }^{5,6}$. UAV systems have the potential to improve the effectiveness of DRER efforts by enhancing first responder capabilities $^{23}$ and providing advanced predictive capabilities and early warning. A wide variety of system types of all sizes with varying capabilities already exist with even more under development.

Disasters or emergencies for which DRER UAV systems could be implemented include: severe storms, tornados, hurricanes, wild fires, tsunamis, floods, earthquakes, avalanches, civil disturbances, oil or chemical spills, and urban disasters.

\section{Systems Analysis Applied to Unmanned DRER Systems}

The optimal design of unmanned systems for DRER applications is dependent not only on easily quantifiable vehicle and mission requirements but also more abstract constraints such as public perception, appropriate levels of vehicle autonomy, and collective system-of-systems interfaces and design. A generalized systems analysis approach will therefore be conceptually outlined for application to this problem with the purpose of finding an optimal system design trade space given a set of requirements and other factors and considerations ${ }^{7}$.

Robotic solutions to large-scale problems generally fall between architecture solutions that have either many small simple systems and a few large complex systems. Finding the optimal design space is a task that involves weighing many factors and carefully analyzing the overall utility of the architecture. Additionally, civil DRER missions are highly interdisciplinary and therefore the degree of reliance on other, perhaps non-aerospace, industries and technologies must be analyzed. In this regards the systems analysis of DRER missions and applications might pose new analytical challenges. 
Many design tradeoffs exist in the realm of unmanned aerospace systems. When these systems are applied to DRER missions, technologies, motivators, and other factors not usually concerning aerospace systems come into play. For example, DRER UAV systems may require a substantial investment in database and communications technology whereas more conventional UAV systems might only require simple radio/satellite links. A further example is the trade-off for smaller versus larger vehicles to minimize collateral damage in the event of failure. Conflict this versus the ability to accommodate large payloads providing advanced capabilities. These tradeoffs do not alter the methodology by which one would approach the problem. However, the key is to correctly identify and assess all trades, especially interdisciplinary trades which may have not previously been associated with aerospace systems.

\section{Case Study - Tornado Alley}

The application of a DRER UAV system to a severe storm, or especially the manifestation of a tornado event, similar to those common throughout the "tornado alley" region of the U.S., will now be discussed. Tornados are violent columns of rotating air, in contact with both the ground and the source storm cells, that pose a significant threat to humans and property ${ }^{14,15,16,18}$. They can range in size from a few feet in diameter to over a mile across and are capable of producing extensive damage as result of the high winds that accompany them. Tornados are produced by strong thunderstorms, called supercells. Supercells are capable of producing damage due to heavy rain, large hail stones, and high winds, even when they do not result in tornado formation. Figure 1 depicts schematic views of a typical supercell thunderstorm. Methods currently exist to detect tornados using modern weather $\operatorname{radar}^{17}$ though coordination with spotters in the field is usually required to achieve more than moderate success and avoid false alarms.

Tornado watch, monitoring, and first response has been selected as a scenario in which UAV assets will likely be beneficial and spawn the development of new technologies. High altitude long endurance (HALE) storm monitoring platforms are currently operational in earth science roles, however, the role of the 'tornado sentinel' mission described here is significantly different. This case study will serve to demonstrate a general set of guidelines concerning the technology development, manufacture, stationing, deployment, and active use of DRER UAV systems. A number of notional technologies will also be proposed which would benefit this application but for which no current widely available practical examples exist.

The primary purpose of utilizing autonomous assets to aid DRER efforts in tornado situations is to save human lives by providing enhanced advanced warning and improving first response.
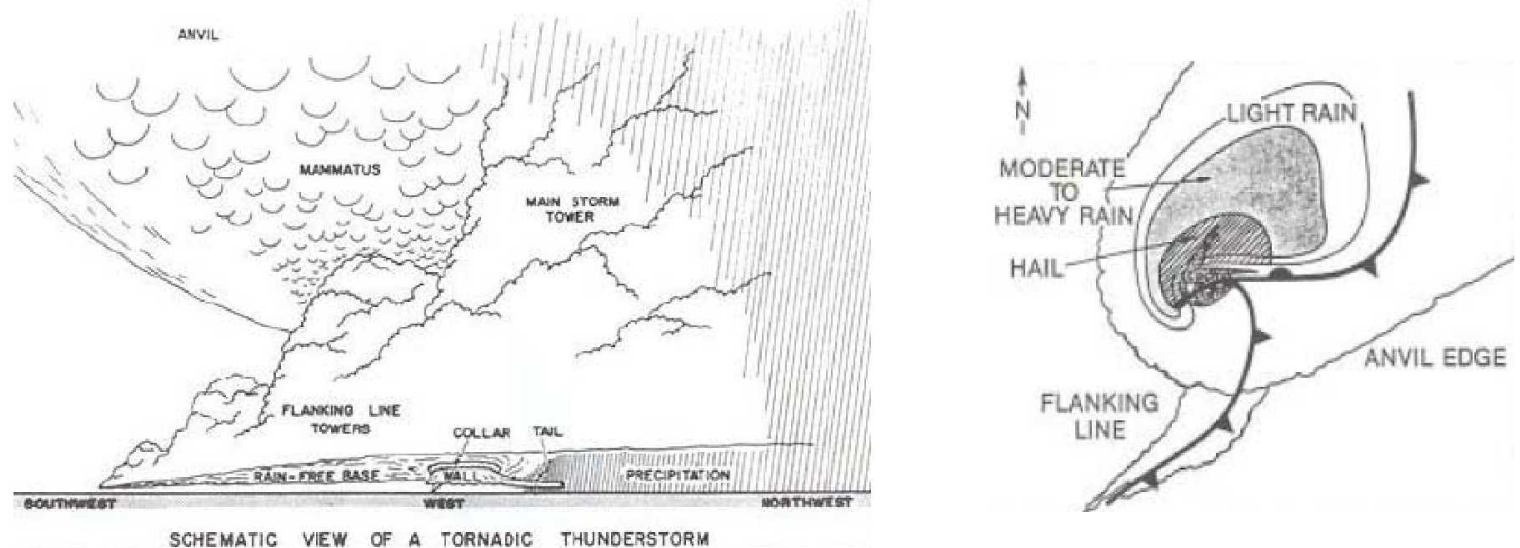

Figure 1: Schematic side and top views of a typical supercell thunderstorm ${ }^{18}$.

\section{A. Essential Technology}

The tornado watch mission would require certain technologies be implemented and could benefit from implementation of new technology. These technologies fall into one of three categories: onboard hardware, onboard software, and ground systems.

\section{i. Hardware Technology}


Onboard hardware technology includes all technologies that involve development and manufacture of new flight hardware for installation onboard the vehicle system itself and also includes technologies related to the design of the flight vehicle itself. A natural area of concentration for an unmanned aerial system is sensors, specifically those for vision ${ }^{19}$. Advanced synthetic vision systems would provide ground operators a clearer picture of developing scenarios and offer a greater depth of vision than even a storm spotter on the ground may be able to achieve. Incorporation of night vision and infra-red spectrum technologies would aid night operations. Onboard radar would increase spatial awareness and improve collision avoidance as well as being a vital asset to the meteorological sensing abilities of the vehicle, as tornado-generating weather patterns can be identified using Doppler radar with a high degree of accuracy. Current micro-radar systems such as MiniSAR are small enough to fly on UAVs weighing only a few hundred pounds, but advances in the near future could put radar systems on smaller aircraft. Improved vision systems are a vital technology because many software technologies will rely on high quality imaging.

Weather monitoring sensors are the primary focus of many earth science aircraft. High quality weather data sensing instruments are important to minimize errors in atmospheric measurements such as temperature, pressure, and humidity. Research into methods for measuring wind velocity from aircraft would contribute to weather monitoring capabilities. The contribution of wind velocity measurements would be more vital when the aircraft is flying near of in severe storms, however this is a region where traditional methods will not yield accurate readings and therefore new technology would be needed. Cloud monitoring is a fundamental aspect of storm and tornado spotting. Sensors capable of distinguishing cloud type would therefore be highly desirable. Given the nature of the composition of clouds (water vapor) such a system might likely be composed of special vision sensing hardware feeding data to image processing software.

Tornados and the storms that generate them are severe weather systems. Flight of manned aircraft near these systems is generally avoided. A tornado watch aircraft designed to fly in such storms, therefore, must be structurally robust and have suitable performance margins and control authority allowing it to maneuver in turbulent, high-wind, high-precipitation, icing-favorable environments. Robust structures, anti-ice, and de-ice systems are essential. High visibility airframe design is another technology concerning the physical aircraft structure. Though it is intended for tornado-watch aircraft to spend a majority of their time aloft in areas most all other aircraft will avoid (near storms), a high visibility vehicle will be easier for other aircraft to spot and avoid during transition phases, test flights, or other clear weather operations.

High visibility airframes could also be considered a component of risk mitigation hardware. Minimizing risk to the public is important to gaining public acceptance as well as adhering to the system's own purpose (to save lives). Technologies such as parachute systems, inflatable 'reserve' wings, and miniature redundant systems could all serve as safety systems in the event of aircraft failures. It can be noted that redundant systems may very well be impractical on small scale UAVs, which is the reason for the desire to miniaturize any redundant backup systems. Smaller backup systems would allow the inclusion of redundancies on smaller size aircraft. The importance of such hardware failsafe systems may be magnified by the severity of the environment in which they are intended to fly. Vehicle health monitoring sensors would be beneficial hardware technology by way of increasing awareness of potential onboard problems during and between flights and therefore reducing the frequency of failures.

\section{ii. Software Technology}

Software technology includes any system or ability that is implemented via computer or other means of processing. The core software ability technology that will drive the tornado watch mission is weather identification, tracking, and forecasting. Data streams from the vehicle's sensors could be fed to onboard software systems or ground based computers to update weather predictions and movement models in real time. Aerial assets with real time weather monitoring systems would provide a better map of severe storm impact areas and assist in issuing severe weather warnings or otherwise alerting the public of potential danger.

Weather prediction capabilities are pre-event and proactive in nature. However, after a tornado or other severe weather strikes, there are notional software technologies that would assist first response personnel. An aerial vehicle could overfly a damaged area and assign it a 'destruction rating,' classifying the degree to which buildings, roads, etc. have been impacted. An associated probability-of-survival for any building collapses could also be determined to assist emergency personnel in concentrating their efforts where they would be most beneficial. To further assist responding vehicles, aerial assets could determine if 
any roads are blocked or damaged and route emergency vehicles around any trouble spots. This would be much like modern driving GPS units with 'alternate route' and 'traffic alert' capabilities but tailored specifically to this scenario and monitored in real time by an on station aerial vehicle.

The meteorological and first responder assistance technologies combine software with the environmental and vision sensors of the aircraft to implement new actions or augment existing capabilities. Other software technologies, however, will imitate the behaviors of manned aircraft to allow for easier integration of new unmanned systems into existing regulated airspace. Technology that would allow an unmanned vehicle to 'talk to' air traffic control would allow it to fly in controlled airspace. Though the notional tornado watch vehicle would not typically fly near other aircraft, the ability to do so would minimize risk to the public in the event it does. An unmanned vehicle equipped with a transponder and in contact with air traffic control could also be designed to respond to orders given by air traffic control. This would provide a measure of safety in a scenarios where UAV-tracked severe weather systems pass near highly populated areas or major airports. Such technology would enable the UAV to avoid all other traffic, minimizing the UAV's impact on current air traffic systems.

\section{iii. Ground Technology}

Ground technologies include technology used by humans on the ground to interface with and utilize data from aerial assets. There is overlap between ground technologies and software technologies. This is primarily because any software technology can be implemented either with onboard computers or ground based computers. Ground based technologies, therefore, can include those weather prediction and air traffic management technologies described earlier.

The core ground technology required is an expanded and enhanced communications and database network. This expanded network serves to both relay information to first responders as well as interface with and supplement public emergency broadcast systems. Data sent to first responders can included automated messages generated by the UAV (as results of software technologies) or raw video streams. Some of this information could be made directly available to the public, but this must be done carefully to avoid initiating panic or infringing on public privacy. An enhanced emergency broadcast system would reach the public with more directive warnings and information than currently exist today. Systems would need to be developed to handle the general public, rural areas, transient areas (freeway, rail, etc.), and large crowds (sporting events, etc.). The emergency alerts would give information tailored to the situation, and would be distributed across many mediums. For example, in the event of a tornado, the system would distribute recommendations as to how to quickly find a safe location to the transient public, so drivers on a highway with no immediate access to storm shelters can take action to get to a safe location. Examples of transmission mediums include portable GPS receivers, radio, satellite radio/communications, cell phones, internet portals, television, public sirens/warning stations, and individual special warning receivers. Special receivers could be developed and made available to the public that are designed to receive emergency alerts. Such receivers would be especially useful in rural areas where other communication mediums do not exist or are limited.

\section{B. Mission Interface}

Autonomous vehicles may fly much of their missions independently, however, they still interface with ground controllers and require operational support. The operational interface and control of a tornado watch vehicle could come from any number of sources. The many disciplines and industries involved in the prediction, monitoring, and response to tornados all must be taken into consideration when developing an interface network for such a vehicle system.

\section{i. Controlling Agency}

The management of a tornado watch vehicle system could be the responsibility of any one of several groups. The National Oceanic and Atmospheric Administration (NOAA) is a likely candidate since they currently manage the issuance of weather watches and warnings in addition to weather prediction across the nation. Both the National Weather Service (NWS) and the NOAA's Aviation Weather Center are branches of the NOAA which currently deal with much of the prediction side of severe weather in the U.S. today. Other likely candidates are law enforcement and emergency response agencies, the national guard and military, or private companies who operate and maintain unmanned vehicle systems. The issue of control will largely depend on where the funding and leadership exists to operate such a system. If advanced communications technology is built into a tornado watch vehicle system, the data from the 
system could be easily and seamlessly be distributed to anywhere it is needed, regardless of who actually controls the intricacies of the vehicle operations.

A note concerning potential control by military entities is that military control may have a benefit to technological advancement. Though this mission is designed to be a civil mission, military involvement may allow for more rapid advancement of technology through the use of military UAV systems since military UAV systems currently fly more routinely and with fewer restrictions than civil UAV systems.

\section{ii. Degree of Control}

The degree of control of an autonomous vehicle can vary from remotely piloted to completely autonomous ${ }^{2,3}$. This level of autonomy is derived from mission requirements, and will influence the level of autonomous data processing power necessary onboard a given vehicle system $^{8,9}$. Once level of autonomy has been determined, there are many other considerations as to how an unmanned tornado watch vehicle will be controlled. It will be noted that the larger, more complex, or more robust a system is, the more expensive and time intensive it will be to manufacture, operate, and maintain. Since great benefit may be obtained from certain increases in complexity, however, decisions would ideally be made based on full cost-benefit analysis of a given system. A notional base for the mission control system for a tornado watch vehicle would be to use weather prediction input for vehicle routing while watching systems or loitering on station, use storm-centered coordinates for maneuvering around developing tornados, and use earth coordinates for flight over first response zones in the wake of a tornado or storm.

Individual vehicle control is only one aspect of the overall mission control scheme. Vehicle deployment strategy is another. Single vehicle operation by independent weather stations may be useful, however multiple vehicles can offer wider coverage areas and redundancy. Multiple vehicles may be flown independently near the same storm, or in a flock operating together ${ }^{13}$. Multiple vehicles based at the same location, or nearby locations may also be used to maintain vehicles on station in the air at all times. Vehicles on station, or staged at strategic points on the ground, would provide faster response times. Such strategies would require vehicles to be stationed at many points throughout the regions where tornados are common. This again goes back to the issue of what agency or group will command the system. If, for example, the national weather service were to command the system, a vehicle asset might be placed at each weather station. Subsequent deployment strategies for a given vehicle distribution system would then be dependant on the desired use, such as pre-event monitoring and intensive weather prediction, or post-event monitoring and more concentration on first responder aid.

Examples of pre-event monitoring include general storm surveillance or advanced warning. Storm surveillance would entail flying patterns ahead of moving fronts and recording weather data useful for predicting areas of heavy rain, hail, and high winds. Advanced tornado warning flight tracks would place vehicles inside the storms with the intent on forecasting and detecting tornados specifically. Vehicles in such flight tracks would generally avoid the high risk (high velocity and conditionally rotating air columns) flight zone near the center of the storm. Post-event monitoring would include all damage assessment and first-responder support roles. Figure 2 provides an overview of notional flight patterns.

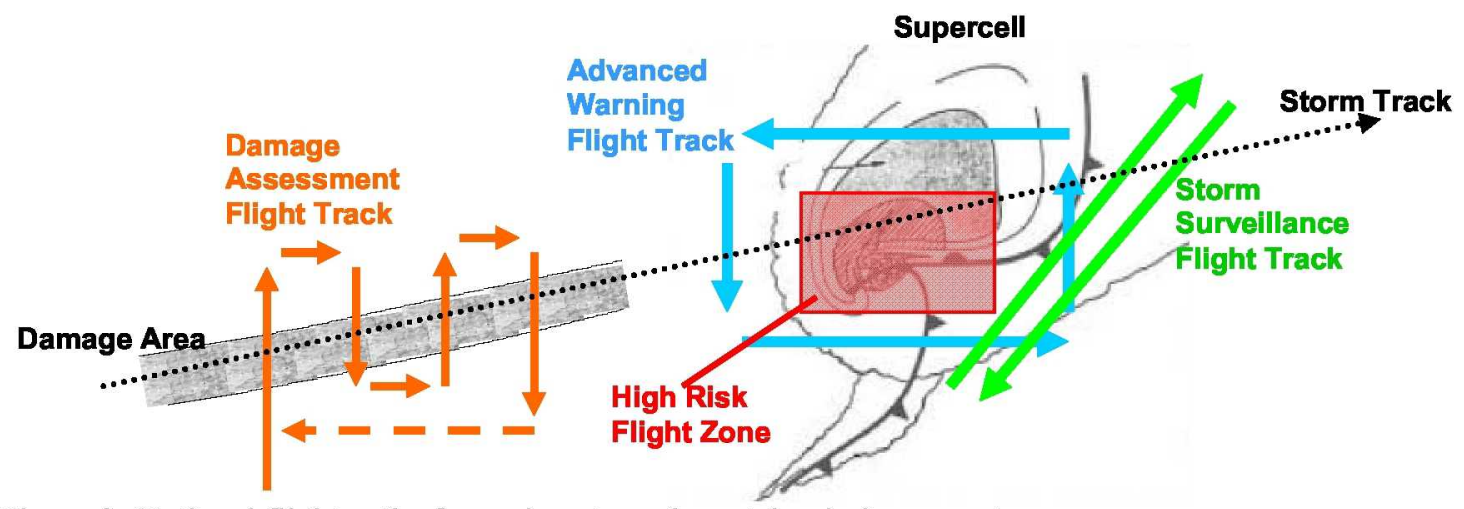

Figure 2: Notional flight paths for various tornado watch mission aspects.

\section{iii. Effect on Existing Systems}


Many systems will be involved or affected to some extent when implementing a new system such as an autonomous tornado watch vehicle system. Autonomous vehicles will be interfacing with ground control stations as well as air traffic control and navigation satellites. Data from the vehicles will be sent to ground units from both weather prediction and emergency response disciplines. Data processing systems will also interface with weather satellites and other weather stations. The general public and any system required to implement an advanced emergency broadcast system will also be involved. Additionally systems relating to the operation and maintenance of the unmanned vehicles themselves will be involved.

\section{Need}

The need for civil UAV assets stems from the need for the government to appear prepared and responsible in the public view. Public safety is a primary concern of the government and the public's ability to 'weather' severe storms would be improved by advanced weather warning and response systems. Increased public awareness of the usefulness of unmanned civil assets would benefit the industry by making the public more accepting of both the concept of aerobots and increased focus on research into experimental unmanned technologies. This increased awareness and acceptance will be an important step towards modifying regulations regarding unmanned vehicles to allow for more routine unmanned flight operations.

\section{Risk}

There is inherent risk to humans when unmanned aerial systems are flown in close proximity to inhabited areas ${ }^{4,12,20}$. High levels of decision-making reliability should be implemented into vehicle autonomy to increase safety by decreasing risk of collision. Several risk mitigation strategies could be implemented to protect against potential system or hardware failures. Vehicle design and size can be tailored to minimize energy transfer in the event of a collision. Smaller vehicles would result in less kinetic energy transfer upon impact and therefore less risk to the public. It is worth noting that as technology advances, vehicle components can be made smaller, therefore allowing for vehicles with a constant sensing capability to be made smaller.

Strategies for risk mitigation already discussed include parachutes, inflatable wings, and redundant systems. Another strategy is to implement vehicle control systems that utilize certain maneuvers to avoid collisions. Maneuvers could include steep turns and climbs or descents, but could also include maneuvers that are not valid in manned aircraft such as intentionally entering into deep stall with the idea that the UAV system has a degree of expendability which can be exploited to avoid collisions and maintain public safety.

\section{E. Cost}

Cost is a key parameter in determining the feasibility of such a civil UAV system. To be accepted into the civil market the individual aerial assets would have to be expendable or maintainable at low cost. Since it is likely that a high degree of robust technology would go into such an asset the cost per vehicle may be greater than what is deemed expendable. To date no comprehensive UAV cost models have been developed, though studies have been done to characterize the market ${ }^{10,11}$. Unfortunately the total cost per flight hour for modern civil unmanned aerial systems currently exceeds that of manned aircraft. There is hope that this will change, however, as new technology develops and applications become more widespread. Even as overall worldwide demand increases the civil UAV market remains a niche market which will be marked by high operational costs in comparison to manned aircraft. The deciding factor will become whether or not the benefits gained from implementation of life-saving UAV systems are worth the price.

\section{F. Design Tradeoff Examples}

The design space of a tornado watch vehicle has many opposing variables which result in design tradeoffs. Here several sample tradeoffs will be discussed.

Complex systems all have non-zero fail rates in the long term. Failure rate increases as complexity increases. Higher complexity can lead to greater sensing and data gathering ability. This increase in utility and its positive public perception, however, is offset by the negative public perception of more common failures.

Tornados are products of powerful storm systems. Manned aircraft avoid such weather systems because they are too turbulent and dangerous to fly in or near. Unmanned aircraft must be designed and 
manufactured to be very resilient to fly in such weather systems. In general, as aircraft resiliency and toughness against flight in harsh weather conditions is improved, aerodynamic performance and efficiency is sacrificed. Flight proximity to a tornado, and therefore data gathering capability, will be governed by flight resiliency. There will be practical limits as to a chosen design point, for example, enough flight performance must be maintained for the vehicle to be able to achieve flight speeds high enough to maintain positive groundspeed even against strong headwinds near a storm, however, at the same time enough flight resiliency must be maintained to prevent loss of control in the highly turbulent wind shear conditions near a storm.

The degree of resiliency of the vehicle could also be contrasted against the degree of expendability of the vehicle. It may be practical to manufacture several 'expendable' vehicles as opposed to fewer, more complex, assets. In such a case, a vehicle with lower resiliency to weather may be able to fly closer to a storm if it is considered expendable.

Response time is of primary concern in the operation of a first-responder aid system. Though severe thunderstorms have life cycles that generally span on the order of hours, tornados can develop, strike, and dissipate on the order of ten minutes. Response time is dependent on vehicle station location and proximity, vehicle readiness, and time to arrive on station. Deployment strategy also has influence, as these variables will change in definition if the vehicle is kept in continuous loiter as opposed to stationed on the ground or some point between continuous aloft and ground based (such as ground based but put on station in loiter under certain weather forecast conditions). The goal of the response time tradeoff is to achieve the smallest possible response time while constrained by total cost, where cost is directly proportional to the number of vehicles used as well as the complexity of the vehicles. Figure 3 presents some sample relationships between design variables which could potentially be used in defining a design space for a tornado watch vehicle system. Note in Figure 3 that storm formation time refers to the development rate of the storm system and time to arrive on station -- related to cruise speed, climb rate, and station location -- is the time it would take a vehicle asset to get from its stationed position to the necessary flight pattern at the storm. Essentially, time to arrive on station determines which type of storms (slow developing or fast developing) a vehicle can successfully respond to.
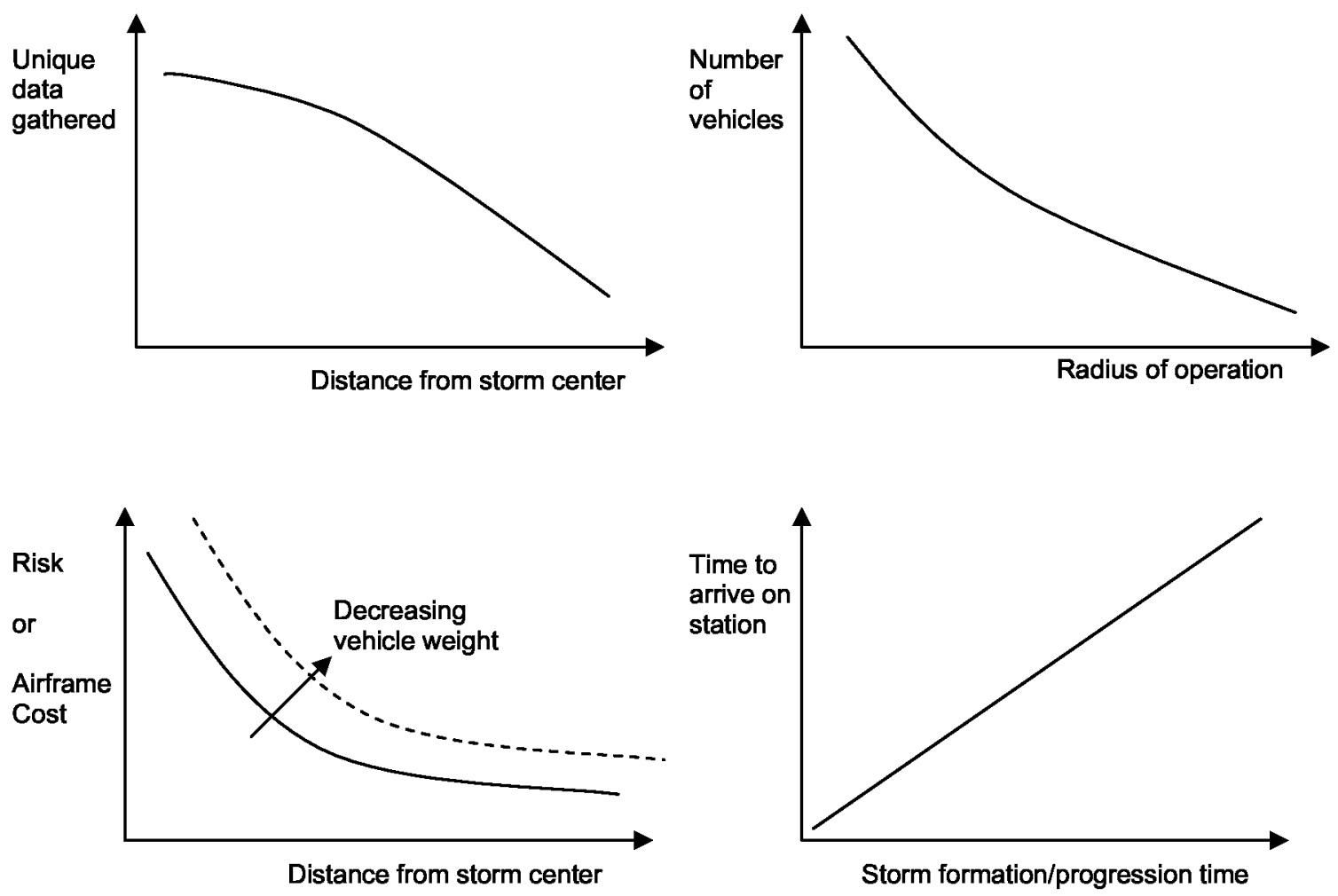

Figure 3: Sample design variable dependency trends. 


\section{Conclusion}

Civil-sector UAV systems have the ability to benefit the public by bolstering DRER efforts in the U.S. advocacy for civil UAV systems and their flight privileges will expand public awareness of the benefits and true nature of the risk of unmanned vehicle operations. A better educated public will be more willing and able to support and fund development of new technologies and implementation of new concepts. Advances in systems technology will enable the advancement and expansion of the UAV market worldwide.

\section{Acknowledgements}

The author would like to acknowledge Larry A. Young of NASA Ames Research Center for his mentoring and contributions to the work presented in this paper.

\section{References}

${ }^{1}$ Young, L. A., "Future Roles for Autonomous Vertical Lift in Disaster Relief and Emergency Response," AHS International Specialists' Meeting on Advanced Rotorcraft Technology and Life Saving Activity, Aichi, Japan, November 15-17, 2006.

${ }^{2}$ Young, L.A., Yetter, J.A., Guynn, M.D., "System Analysis Applied to Autonomy: Application to High-Altitude Long-Endurance Remotely Operated Aircraft," AIAA Infotech@Aerospace Conference, Arlington, VA, September 2005.

${ }^{3}$ Young, L.A., "System Analysis Applied to Autonomy: Application to Human-Rated Lunar/Mars Landers," AIAA Space 2006 Conference, San Jose, CA, September 19-21, 2006.

4 Young, L.A., "Aerobots as a Ubiquitous Part of Society," AHS Vertical Lift Aircraft Design (VLAD) Conference, San Francisco, CA, January 18-20, 2006.

5 Young, L.A., "Enhanced Rescue Lift Capability," AHS International $63^{\text {rd }}$ Annual Forum, Virginia Beach, VA, May 1-3, 2007.

${ }^{6}$ Aiken, E.W., Ormiston, R.A., Young, L.A., "Future Directions in Rotorcraft Technology at Ames Research Center," AHS 56 ${ }^{\text {th }}$ Annual Forum, Virginia Beach, VA, May 2-4, 2000.

7 Young, L.A., "Systems Engineering: When Knowledge and Technology Are the Product," AHS $64^{\text {th }}$ Annual Forum, Montreal, Canada, April 29 - May 1, 2008.

${ }^{8}$ Young, L.A., "Feasibility of Turing-Style Tests for Autonomous Aerial Vehicle 'Intelligence'," AHS International Specialists' Meeting on Unmanned Rotorcraft, Chandler, AZ, January 23-25, 2007.

${ }^{9}$ Freed, M., Harris, R., Shafto, M.G., "Human-Interaction Challenges in UAV-Based Autonomous Surveillance," American Association for Artificial Intelligence Spring Symposium, Palo Alto, CA, March 21-23, 2004.

10 Valerdi, R., "Cost Metrics for Unmanned Aerial Vehicles," AIAA Infotech@Aerospace Conference, Arlington, VA, September 26-29, 2005

11 Papadales, B., Downing, M., "UAV Science Missions: A Business Perspective," AIAA Infotech@Aerospace Conference, Arlington, VA, September 26-29, 2005.

12 Pisanich, G., et. al. "Actions, Observations, and Decision-Making: Biologically Inspired Strategies for Autonomous Aerial Vehicles," AIAA $42^{\text {nd }}$ Aerospace Sciences Meeting and Exhibit, Reno, NV, January 5-8, 2004.

13 Young, L.A., Pisanich, G., "Aerial Explorers and Robotic Ecosystems," International Conference on Computing, Communications and Control Technologies: CCCT'04, The International Institute of Informatics and Systemics, Austin, TX, August 14-15, 2004.

${ }^{14}$ Doswell III, C.A., "Historical Overview of Severe Convective Storms Research," Electronic Journal of Severe Storms Meteorology, January 3, 2007.

${ }^{15}$ Doswell III, C.A., Schultz, D.M., "On the Use of Indicies and Parameters in Forecasting Severe Storms," Electronic Journal of Severe Storms Meteorology, November 5, 2006.

${ }^{16}$ Edwards, R., Lemon, L.R., "Proactive or Reactive: The Severe Storm Threat to Large Event Venues," American Meteorological Society $21^{\text {st }}$ Conference on Severe Local Storms, San Antonio, TX, August 12-16, 2002.

${ }^{17}$ Mitchell, E.D., et. al., "The National Severe Storms Laboratory Tornado Detection Algorithm," American Meteorological Society Journal, Volume 13, Issue 2, pp. 352-366, June 1998.

${ }^{18}$ Doswell III, C.A., et. al. "Advanced Spotters' Field Guide," US Department of Commerce, 2005. 
19 Kontitsis, M., Valavanis, K.P., Tsourveloudis, N., "A UAV Vision System for Airborne Surveillance," IEEE International Conference on Robotics \& Automation, New Orleans, LA, April, 2004.

${ }^{20}$ Weibel, R.E., Hansman Jr., R.J., "Safety Considerations for Operation of Different Classes of UAVs in the NAS," AIAA $3^{\text {rd }}$ Unmanned Unlimited Technical Conference, Chicago, IL, September 20-22, 2004.

${ }^{21}$ Cox, T.H., et. al., "Civil UAV Capability Assessment," NASA internal technical report, December 2004.

${ }^{22}$ Whalley, M., et. al., "Design, Integration, and Flight Test Results for an Autonomous Surveillance Helicopter," AHS UAV Specialists Meeting, January 19, 2005.

${ }^{23}$ DeBusk, W.M., "Daughtership Release Flight Dynamics of the Aerial Surveyor Hybrid Autonomous Aircraft," AHS Aeromechanics Specialists Meeting, San Francisco, CA, January 23-25, 2008. 